\title{
NeuroAiD: Properties for Neuroprotection and Neurorepair
}

\author{
C. Heurteaux ${ }^{\mathrm{a}}$ C. Widmann ${ }^{\mathrm{a}} \quad$ H. Moha ou Maati ${ }^{\mathrm{a}} \quad$ H. Quintard ${ }^{\mathrm{a}} \mathrm{b} \quad$ C. Gandin ${ }^{\mathrm{a}}$ \\ M. Borsotto ${ }^{a} \quad J . V^{2}$ eyssiere ${ }^{a} \quad$ B. Onteniente ${ }^{c}$ M. Lazdunski ${ }^{a}$ \\ a Institut de Pharmacologie Moléculaire et Cellulaire, Centre National de la Recherche Scientifique (CNRS), \\ Université Nice Sophia Antipolis, Valbonne, ${ }^{b}$ Centre Hospitalo-Universitaire de Nice, Hôpital St Roch, Nice, and \\ 'INSERM UMR861, Université d'Evry, Paris, France
}

\section{Key Words}

Stroke - Cardiac arrest - MLC601 - MLC901 - Neuroprotection · NeuroAiD · Brain plasticity $\cdot$ Neurogenesis

\begin{abstract}
Background: Treatments for stroke and other brain injuries are limited. NeuroAiD has been shown to be beneficial in clinical studies. We reviewed the pharmacological effects of NeuroAiD on the normal and ischemic brain and neurons. Methods: In vivo and in vitro experiments using mouse model of stroke (focal ischemia), rat model of cardiac arrest (global ischemia) and cortical neurons in culture were reviewed and summarized. Results: NeuroAiD improved survival, attenuated infarct size, improved functional recovery in the model of focal ischemia, and protected neurons against glutamate-induced injury. Furthermore, it enhanced cognitive recovery by reducing hippocampal CA1 cell degeneration, DNA fragmentation, Bax expression and malondialdehyde release in the model of global ischemia. Activation of the Akt survival pathway and opening of $\mathrm{K}_{\text {ATP }}$ channels may contribute to the neuroprotective properties of NeuroAiD. NeuroAiD increased BDNF expression and induced proliferation of cells which differentiate and mature into neurons. It enhanced rosette formation of human embryonic stem cells. NeuroAiD-treated embryonic cortical neurons developed into neurons with longer neurites, dens-
\end{abstract}

er outgrowths and networks, and more synaptic release sites. Conclusions: NeuroAiD demonstrated both neuroprotective and neuroregenerative properties in rodent models of focal and global ischemia and in cortical cell cultures. These properties would be important for developing a treatment strategy in reducing the long-term disability of stroke, cardiac arrest and other brain injuries.

Copyright $\odot 2013$ S. Karger AG, Basel

\section{Introduction}

Despite decades of academic and industrial research, stroke continues to defy attempts of developing effective treatments. Thrombolysis within 3-5 h of stroke onset remains the most effective treatment for acute ischemic stroke. However, recanalization of the occluded vessel with thrombolytics is restricted to only a small proportion of patients due to a short window of treatment opportunity and several contraindications. In the past 2 decades, numerous clinical trials have failed to demonstrate a benefit in treating cerebral ischemia. Although many targets have been pursued, including antioxidants, calcium channel blockers, glutamate receptor blockers and neurotrophic factors, there is no molecule yet able to clinically induce effective brain protection. The need for alternative therapeutic strategies is high.

\section{KARGER}

E-Mail karger@karger.com www.karger.com/ced

\section{(c) 2013 S. Karger AG, Basel} 1015-9770/13/0357-0001 $\$ 38.00 / 0$

Karger

Open access

This is an Open Access article licensed under the terms of the Creative Commons Attribution-NonCommercial-NoDerivs 3.0 License (www.karger.com/OA-license), applicable to the online version of the article only. Distribution for non-commercial purposes only.
Prof. M. Lazdunski

Institut de Pharmacologie Moléculaire et Cellulaire

Centre National de la Recherche Scientifique (CNRS), Université Nice Sophia Antipolis 660 Route des Lucioles, FR-06560 Valbonne (France)

E-Mail lazdunski@ipmc.cnrs.fr 
Neuroprotection refers to the strategies and relative mechanisms able to defend the central nervous system against neuronal injury due to acute neuronal disorders (such as stroke and cardiac arrest) but also to chronic neurodegenerative disorders (such as Alzheimer and Parkinson diseases). On the other hand, neurogenesis and angiogenesis are key mechanisms of recovery after stroke [1]. Ideally, therapeutic agents against stroke should be able to display multiple effects in impeding the ischemic cascade propagating from the core to the penumbra as well as in stimulating proliferation and differentiation of new neural cells to repair. Herbal medicine may represent a valuable resource in search of effective therapeutics against ischemia. Traditional Chinese medicine (TCM) is one of the world's oldest documented medical systems based on herbal medicines. It has been successfully used for centuries to treat a wide variety of ailments and has recently attracted increasing attention from both industry and academia. Most traditional therapeutic formulations consist of a combination of several plants. The combination of multiple herbal components is thought to maximize therapeutic efficacy by facilitating synergistic actions and ameliorating or preventing potential adverse effects while at the same time aiming at multiple targets.

In the search of drugs with both neuroprotective and recovery-enhancing properties, MLC601 and MLC901 represent particularly interesting candidates. MLC601 (NeuroAiD, Moleac Pte. Ltd., Singapore) is a TCM which was first registered by the Sino Food and Drug Administration in 2001 after being evaluated in clinical trials in China as a drug to facilitate recovery after stroke [2]. It combines 9 herbal components (radix astragali, radix salviae mitorrhizae, radix paeoniae rubrae, rhizoma chuanxiong, radix angelicae sinensis, Carthamus tinctorius, Prunus persica, radix polygalae and rhizoma acori tatarinowii) and 5 animal components (including Hirudo, Eupolyphaga seu Steleophaga, calculus bovis artifactus, Buthus martensii and Cornu saigae tataricae). MLC601 treatment is currently used in several countries both in Asia and in the Middle East for stroke patients. In Europe, a simplified formulation (MLC901) consisting of the 9 herbal components is available. It can be used on top of usual medications, including antiplatelets or anticoagulants. It does not seem to have significant side effects [3]. A multicenter clinical trial, called Chinese Medicine MLC601 Efficacy on Stroke recovery (CHIMES), is ongoing in Asia [4]. In parallel, preclinical research on MLC601 and MLC901 has recently started to explore their mechanisms of action in relation to neuroprotection and neurorepair in animal models, as well as at the cellular and molecular levels. MLC601 and MLC901 have been shown to be equivalent in properties in these experiments, so we shall refer mainly to results on experiments on MLC901 in this review.

\section{Neuroprotective Effects}

Consistent with observations of MLC601 efficacy in humans, pharmacological data obtained in rodents have established that MLC901 prevents death of threatened neuronal tissues, decreases cognitive deficits and improves functional outcome by restoring neuronal circuits [5, 6] (fig. 1).

Using the classical mouse model of focal ischemia induced by transient ( $60 \mathrm{~min}$ ) middle cerebral artery occlusion for $60 \mathrm{~min}$, Heurteaux et al. [5] demonstrated that MLC901 treatment, when administered in pre- or posttreatments, improved animal survival as well as functional neurological recovery and decreased neurodegeneration without affecting physiological parameters. An acute intraperitoneal administration of MLC901 ( $1 \mu \mathrm{g} /$ mouse in a bolus of $500 \mu \mathrm{l}$ ) induced a high survival rate and drastically decreased cerebral infarction with a reduction of the stroke volume by around 50\% compared to control ischemic mice at $30 \mathrm{~h}$ after ischemia. MLC901 is also effective in prevention, since a 6-week pretreatment of MLC901 administered in the drinking water $(6 \mathrm{mg} / \mathrm{ml})$ before the induction of ischemia induced a marked reduction of the mortality of treated animals as well as a decrease of their cerebral infarcts.

In the rat model of global ischemia induced by fourvessel occlusion for $20 \mathrm{~min}$ [7], which mimics sudden cardiac arrest and cardiopulmonary resuscitation in humans, MLC901, when intraperitoneally administered in

Fig. 1. Summary of the neuroprotective properties of NeuroAiD in two models of cerebral ischemia (focal and global) and in a model of oxygen glucose deprivation. a MLC901 posttreatment $(0.074$ $\mathrm{mg} / \mathrm{ml}$ ) protects hippocampal CA1 neurons against $30 \mathrm{~min}$ global ischemia and decreases the cognitive deficits in the Morris water maze task. b MLC901 posttreatment $(1 \mu \mathrm{g} / \mathrm{ml})$ decreased the infarct volumes in mice subjected to 1-hour reversible middle cerebral artery occlusion. c Neuroprotective effect of MLC901 on neuronal death induced by 2 -hour oxygen glucose deprivation and its inhibition by glibenclamide, an inhibitor of $\mathrm{K}_{\mathrm{ATP}}$ channels. $\mathbf{d}$ Activation of $\mathrm{K}_{\mathrm{ATP}}$ channels (Kir6.2/SUR1) induced by MLC901 in Xenopus oocytes. Typical current traces recorded in control conditions $(90 \mathrm{~K})$ in the presence of azide $(3 \mathrm{mM})$, which mimics ischemia by reducing the intracellular ATP level, or azide + MLC901 $(1 \mu \mathrm{g} / \mathrm{ml})$. Some images in the figure were reproduced in part with permission from $[5,6,9]$. 

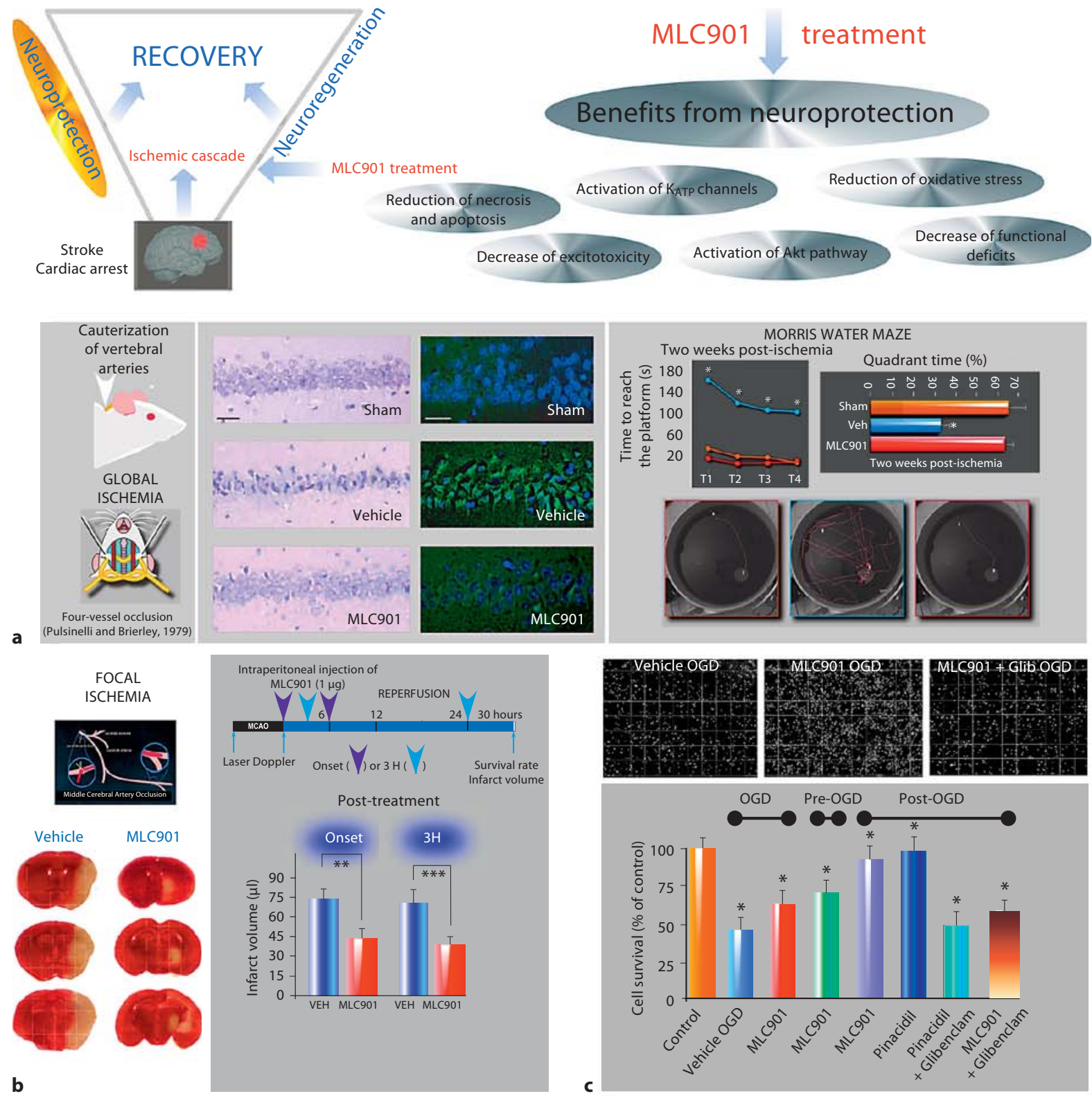

b

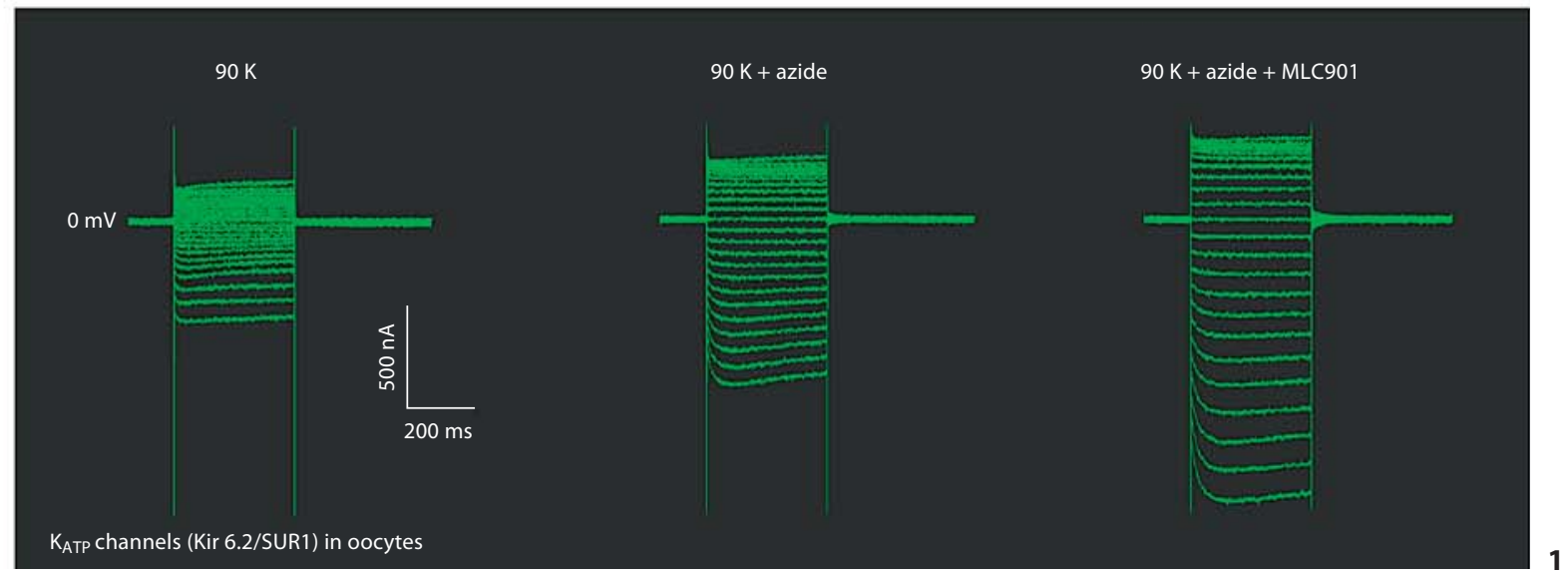


posttreatment $(0.074 \mathrm{mg} / \mathrm{ml}$ in a bolus of $500 \mu \mathrm{l} / \mathrm{rat})$ for 7 days, strongly reduced delayed necrotic and apoptotic neuron death in the vulnerable hippocampal CA1 field after 1 week of reperfusion [6]. Very interestingly, MLC901 provides protection from both focal and global ischemia in rodents when given as late as $3 \mathrm{~h}$ after ischemia.

The improvement of the neurological function impaired by ischemia confirmed the pharmacological efficiency of MLC901. Indeed, MLC901 protection was accompanied in surviving animals by a decrease of behavioral deficits in both models (focal and global) of ischemia, in line with the first promising clinical results of MLC601 efficiency on functional recovery after stroke [2, 8]. MLC901 improved motor performances measured in the accelerated rotarod, actimeter and Morris water maze tests, considered as useful operant conditioning procedures to assess long-lasting deficits after ischemia [5]. Three and 7 days after focal ischemia, MLC901-treated mice showed a significant improvement of their performances on the rotarod and the actimeter compared with the vehicle-treated ischemic group. The functional improvement induced by MLC901 was significantly correlated to the decrease of infarction volume. MLC901 also improved functional recovery after global ischemia as assessed by the Morris water maze and grip strength tests. MLC901 reduced the increase in escape latency and in swim distance induced by ischemia and improved postischemic grip strength [6].

Neurons are extremely vulnerable to hypoxic and excitotoxic injuries. The efficacy of MLC901 has also been demonstrated in a model of oxygen glucose deprivation, which mimics the rapid depletion of oxygen and glucose seen under ischemic conditions in vivo. Deprivation of oxygen and glucose for $2 \mathrm{~h}$ on cortical neurons induces immediate neuronal swelling, followed by calcium influx and neuronal degeneration over the next $24 \mathrm{~h}$, accompanied by release of lactate dehydrogenase. MLC901 decreased exaggerated $\mathrm{Ca}^{2+}$ influx and subsequently attenuated oxygen glucose deprivation-induced excitotoxicity [9]. It has been shown in parallel that MLC901 also induced a strong protective effect against glutamate-induced cell death, an effect that was maintained $24 \mathrm{~h}$ after the excitotoxic injury on cortical neurons in culture [5].

Brain injury following ischemia results from the complex interplay of multiple pathways including excitotoxicity, acidosis, ionic imbalance, peri-infarct depolarization, oxidative stress, inflammation and apoptosis [10]. It was therefore expected that the beneficial effects of MLC901 would be due to 'multitarget' effects.
First, the neuroprotective effect of MLC901 is associated with a large hyperpolarization which is antagonized by glibenclamide, the specific inhibitor of ATP-sensitive $\mathrm{K}^{+}$channels $\left(\mathrm{K}_{\mathrm{ATP}}\right)$ [9]. Activation of $\mathrm{K}_{\mathrm{ATP}}$ channels is a key step in the beneficial effects of ischemic preconditioning [11] and has been proposed as a way to be neuroprotective against brain ischemia [11-15]. Electrophysiological experiments on mouse cortical neurons have demonstrated that MLC 901 acts as an activator of $\mathrm{K}_{\mathrm{ATP}}$ channels as potent as pinacidil, a classical $\mathrm{K}_{\mathrm{ATP}}$ channel opener [9]. This effect was confirmed by the demonstration that coexpression of SUR1 and Kir6.2, the two main subunits of the neuronal $\mathrm{K}_{\mathrm{ATP}}$ channel [16], leading to the potassium channel expression, was potently activated by MLC901. Hyperpolarization induced by MLC901 due to $\mathrm{K}_{\mathrm{ATP}}$ channel activation, particularly in neurons that have suffered from energy deprivation, prevented for a short period the massive release of excitotoxic glutamate and the glutamate-triggered $\mathrm{Ca}^{2+}$ influx $[5,9]$.

In addition to $\mathrm{K}_{\mathrm{ATP}}$ channel activation as a key event in the neuroprotective effect of MLC901, in vivo experiments have shown that MLC901 treatment (in the model of global ischemia) activated in the vulnerable brain regions the serine/threonine kinase Akt (protein kinase B) pathway, which is a central mediator in signal transduction pathways involved in cell survival after cerebral ischemia [17].

The positive therapeutic MLC901 effects are also associated with a decrease of the level of the Bax protein, which is a potent proapoptotic molecule, a member of the Bcl-2 family triggering activation of terminal caspases. This is paralleled by a reduction of TUNEL labeling (a marker of DNA degradation) suggesting that the neuroprotection induced by MLC901 involves a decrease of apoptotic pathways [6].

Reduction of free radicals in the ischemic tissue has long been considered to be one of the potential neuroprotective strategies for limiting the extent of brain tissue damage following ischemia. The level of peroxidation caused by free radical release in the hippocampus of animals undergoing global ischemia, as assessed by the level

Fig. 2. Summary of the neuroregenerative properties of NeuroAid. a MLC901 pretreatment induced neurogenesis and cell proliferation. b MLC901-induced neurogenic effects on human ESC-derived progenitors. c MLC901 pretreatment increased the expression of the neurotrophic factor BDNF in hippocampal CA1 neurons. d MLC901 promotes neurite outgrowth and increases the expression of doublecortin, synaptotagmin and BDNF expression in cortical neurons in cultures. Some images in the figure were reproduced in part with permission from [5].
Heurteaux et al. 

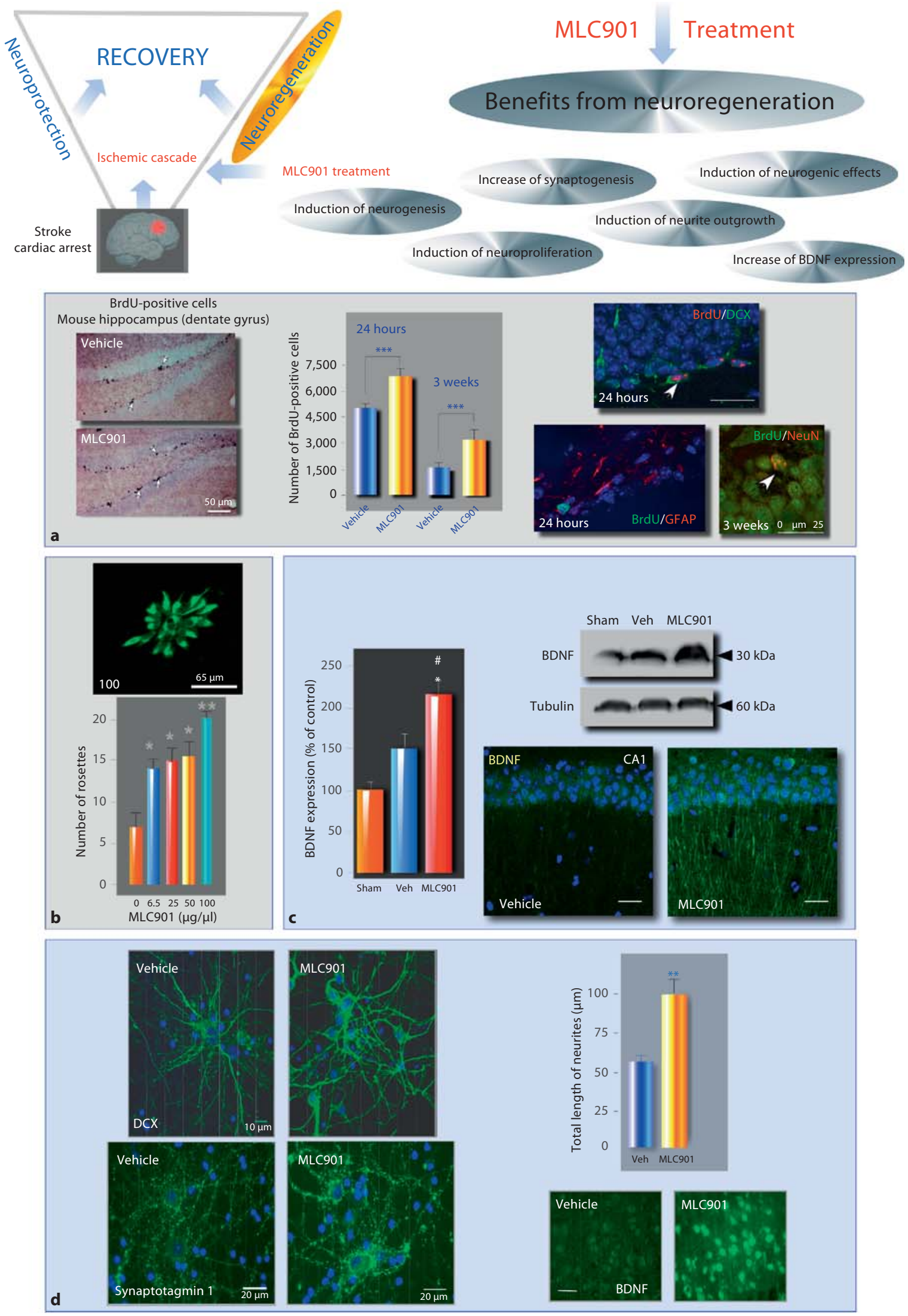
of malondialdehyde, a stable metabolite of the lipid peroxidation cascade and an indicator of cellular oxidation status, was indeed enhanced [18], but this ischemiainduced malondialdehyde production was drastically decreased by a treatment with MLC901. This result indicates that MLC901 also contains substances active as antioxidants [6]. Figure 1 gives a summary of the neuroprotective properties of NeuroAiD.

\section{Neuroregenerative Properties}

Recovery from brain tissue damage depends on effective stimulation of neuroregeneration processes (fig. 2). Therefore, an important question was to know whether MLC901 stimulates neuroplasticity and neurogenesis, thus contributing to optimal recovery of brain function. It is now well known that after ischemia the brain uses its complement of neural plastic responses to reorganize, at least partially, the cortical maps [19]. Changes in cortical organization also include an increase in the number and density of dendrites and synapses. We have shown that MLC901 was able to promote basal neurogenesis. MLC901 treatment in the drinking water for 6 weeks enhanced by 2 -fold the number of newborn cells, which differentiate into mature neurons in 3 weeks [5]. MLC901 has also been shown to stimulate neurogenesis in the subgranular zone of dentate gyrus of rats subjected to global ischemia [6]. MLC901 treatment administered $3 \mathrm{~h}$ after ischemia followed by 1 injection per day for 7 days after reperfusion highly improved the increase in the number of BrdU-positive neuronal precursors after ischemia compared to ischemic vehicle-treated animals. In addition, MLC901-induced neurogenic processes in cortical neurons have also been observed in human embryonic stem cells. MLC901 was shown to have a positive effect on the number of neural progenitors derived from human embryonic stem cells [5]. All these observations taken together suggest that MLC901 contains key molecules that are able to create a neurogenic niche and enriched microenvironment to promote amplification and differentiation of neural progenitors.

In vitro experiments on cultured cortical cells have shown that MLC901 helps to develop a dense axonal and dendritic arborization, illustrated by a large increase of doublecortin fluorescent labeling intensity as well as an enhanced neurite outgrowth. MLC901-treated cortical neurons developed a denser neuritic network with more frequent elongating neurites and branching, resulting in an increased expression of the growth-associated protein
GAP43 in neurite [5]. This neuroregenerative effect of MLC901 is correlated with an increase of synaptogenesis [5], visualized by the increase of synaptotagmin-1 expression, one of the synaptic vesicle proteins having a critical role in synaptogenesis and synapse function [20,21]. All these results highly suggest that MLC901, by its ability to promote neurogenesis, neurite outgrowth and synaptogenesis, has a potential to amplify the intrinsic brain properties for neuroplasticity, favoring subsequent neurological recovery after ischemia.

One possible mechanism of the previously described MLC901 effect includes its ability to stimulate the secretion of BDNF which is an important growth factor regulating neuronal survival [22] and brain plasticity [23]. In vitro data showed that a 6-week MLC901 treatment $(6 \mathrm{mg} / \mathrm{ml})$ increased BDNF expression in cortical neurons [5]. Figure 2 gives a summary of the neuroregenerative properties of NeuroAid.

\section{Conclusions}

(1) NeuroAiD (MLC601/MLC901) has been shown in in vitro and in animal models to have properties consistent with a capacity to neurorepair. This provides scientific support to the present (traditional) use of this therapeutic compound which is most often administered to human patients weeks or months after stroke; (2) NeuroAiD has also been shown to be neuroprotective which strongly suggests that positive effects should be expected for early (tens of minutes, hours) administration after stroke or cardiac arrest, and (3) NeuroAiD displays this variety of beneficial properties in vitro and in animal models because, most probably, it contains a cocktail of active components working on several neuroprotective/ neurorepair mechanisms, some of which have been identified.

\section{Disclosure Statement}

M.L.: Vice President Research Moleac. This work was fully supported by a Research CNRS-Moleac contract: C.H., C.W., H.M.M., H.Q., C.G., M.B., J.V. and B.O. DOI: $10.1159 / 000346228$
Heurteaux et al. 


\section{References}

1 Zhang RL, Zhang ZG, Chopp M: Ischemic stroke and neurogenesis in the subventricular zone. Neuropharmacology 2008;55:345-352.

$>2$ Chen C, Venketasubramanian N, Gan RN, Lambert C, Picard D, Chan BP, Chan E, Bousser MG, Xuemin S: Danqi Piantang Jiaonang (DJ), a traditional Chinese medicine, in poststroke recovery. Stroke 2009;40: 859-863.

-3 Gan R, Lambert C, Lianting J, Chan ES, Venketasubramanian N, Chen C, Chan BP, Samama MM, Bousser MG: Danqi Piantan Jiaonang does not modify hemostasis, hematology, and biochemistry in normal subjects and stroke patients. Cerebrovasc Dis 2008;25: 450-456.

4 Venketasubramanian N, Chen CL, Gan RN, Chan BP, Chang HM, Tan SB, Picard D, Navarro JC, Baroque AC 2nd, Poungvarin N, Donnan GA, Bousser MG: A double-blind, placebo-controlled, randomized, multicenter study to investigate Chinese medicine NeuroAiD efficacy on stroke recovery (CHIMES study). Int J Stroke 2009;4:54-60.

$\checkmark 5$ Heurteaux C, Gandin C, Borsotto M, Widmann C, Brau F, Lhuillier M, Onteniente B, Lazdunski M: Neuroprotective and neuroproliferative activities of NeuroAiD (MLC601, MLC901), a Chinese medicine, in vitro and in vivo. Neuropharmacology 2010; 58:987-1001.

6 Quintard H, Borsotto M, Veyssiere J, Gandin C, Labbal F, Widmann C, Lazdunski M, Heurteaux C: MLC901, a traditional Chinese medicine, protects the brain against global ischemia. Neuropharmacology 2011;61:622631.
7 Pulsinelli WA, Brierley JB: A new model of bilateral hemispheric ischemia in the unanesthetized rat. Stroke 1979;10:267-272.

$>8$ Siow CH: NeuroAiD in stroke recovery. Eur Neurol 2008;60:264-266.

$\checkmark 9$ Moha Ou Maati H, Borsotto M, Chatelain F, Widmann C, Lazdunski M, Heurteaux C: Activation of ATP-sensitive potassium channels as an element of the neuroprotective effects of the traditional Chinese medicine MLC901 against oxygen glucose deprivation. Neuropharmacology 2012;63:692-700.

10 Doyle KP, Simon RP, Stenzel-Poore MP: Mechanisms of ischemic brain damage. Neuropharmacology 2008;55:310-318.

11 Heurteaux C, Lauritzen I, Widmann C, Lazdunski M: Essential role of adenosine, adenosine A1 receptors and ATP-sensitive $\mathrm{K}^{+}$ channels in cerebral ischemic preconditioning. Proc Natl Acad Sci USA 1995;92:46664670.

12 Blondeau N, Plamondon H, Richelme C, Heurteaux C, Lazdunski M: K(ATP) channel openers, adenosine agonists and epileptic preconditioning. Neuroscience 2000;100: 465-474.

13 Heurteaux C, Bertaina V, Widmann C, Lazdunski $\mathrm{M}: \mathrm{K}^{+}$channel openers prevent global ischemia-induced expression of c-fos, c-jun, heat shock protein, and amyloid beta-protein precursor genes and neuronal death in rat hippocampus. Proc Natl Acad Sci USA 1993 90:9431-9435.

14 Lauritzen I, De Weille JR, Lazdunski M: The potassium channel opener (-)-cromakalim prevents glutamate-induced cell death in hippocampal neurons. J Neurochem 1997;69: 1570-1579.
15 Liss B, Roeper J: Molecular physiology of neuronal K-ATP channels (review). Mol Membr Biol 2001;18:117-127.

16 Inagaki N, Gonoi T, Clement JP 4th, Namba $\mathrm{N}$, Inazawa J, Gonzalez G, Aguilar-Bryan L, Seino S, Bryan J: Reconstitution of IKATP: An inward rectifier subunit plus the sulfonylurea receptor. Science 1995;270:1166-1170.

17 Franke TF, Kaplan DR, Cantley LC: PI3K: downstream AKTion blocks apoptosis. Cell 1997;88:435-437.

18 Friberg H, Wieloch T, Castilho RF: Mitochondrial oxidative stress after global brain ischemia in rats. Neurosci Lett 2002;334:111114.

19 Chopp M, Li Y, Zhang ZG: Mechanisms underlying improved recovery of neurological function after stroke in the rodent after treatment with neurorestorative cell-based therapies. Stroke 2009;40:S143-145.

20 Sudhof TC: The synaptic vesicle cycle: a cascade of protein-protein interactions. Nature 1995;375:645-653.

21 Jessell TM, Kandel ER: Synaptic transmission: a bidirectional and self-modifiable form of cell-cell communication. Cell 1993;72 (suppl):1-30.

22 Mattson MP: Glutamate and neurotrophic factors in neuronal plasticity and disease. Ann NY Acad Sci 2008;1144:97-112.

23 Volosin M, Song W, Almeida RD, Kaplan DR, Hempstead BL, Friedman WJ: Interaction of survival and death signaling in basal forebrain neurons: roles of neurotrophins and proneurotrophins. J Neurosci 2006;26:7756-7766. 